\title{
Usefulness of surgical lung biopsies after cryobiopsies when pathological results are inconclusive or show a pattern suggestive of a nonspecific interstitial pneumonia
}

\author{
Benjamin Bondue ${ }^{1 *}$ D, Dimitri Leduc ${ }^{1}$, Antoine Froidure ${ }^{2}$, Thierry Pieters ${ }^{2}$, Olivier Taton ${ }^{1}$, Vincent Heinen ${ }^{3}$,
}

Patrick Alexander ${ }^{4}$, Delphine Hoton ${ }^{5}$, Florence Dome ${ }^{6}$ and Myriam Remmelink ${ }^{7}$

\begin{abstract}
Background: Although increasing data supports the use of transbronchial lung cryobiopsies (TBLCs) for the diagnosis of diffuse parenchymal lung diseases (DPLDs), its role as an alternative to surgical lung biopsy (SLB) is still under debate. The aim of this study was to assess the benefit of additional SLBs performed in selected patients after TBLCS.

Method: We conducted a multicentric Belgian prospective trial in which SLBs were performed after TBLCs when the pathological diagnosis was uncertain or if a nonspecific interstitial pneumonia (NSIP) pattern was observed hypothesizing that SLB could provide additional information and that a co-existent UIP pattern could be missed.

Results: Eighty-one patients with TBLCs performed for a DPLD were included in the study between April 2015 and December 2019. A specific histological diagnosis was obtained in 52 patients (64\%) whereas no pathological diagnosis following TBLCs was obtained in 13 patients (16\%) and a pattern suggestive of a NSIP was observed in 16 patients (20\%). Fourteen out of these 29 patients had SLBs after TBLCs. SLBs showed a UIP pattern in 11 (79\%), a pattern suggestive of a hypersensitivity pneumonitis in two (14\%) and a NSIP pattern in one patient (7\%). Among the 16 patients with pathological NSIP following TBLCs, six underwent a SLBs showing a UIP in five and confirming a NSIP in one patient only. A retrospective pathological analysis of patients having both procedures showed a lower diagnostic confidence and agreement among pathologists for TBLCs compared to SLBs. Major factors underlying the added value of SLBs were the bigger size of the sample as well as the subpleural localization of the biopsies.
\end{abstract}

Conclusions: TBLCs are useful in the setting of DPLDs with a good diagnostic yield. However, our study suggests that SLB provides critical additional information in case TBLCS are inconclusive or show a pattern suggestive of a NSIP, questioning the accuracy of TBLC to adequately identify this histological pattern.

Keywords: Cryobiopsy, Surgical lung biopsy, Interstitial lung disease, Diffuse parenchymal lung disease, Transbronchial lung cryobiopsy, Nonspecific interstitial pneumonia, NSIP, Idiopathic pulmonary fibrosis, IPF

\footnotetext{
* Correspondence: benjamin.bondue@erasme.ulb.ac.be

'Department of Pneumology, Hôpital Erasme, Université libre de Bruxelles, 808 route de Lennik, 1070 Brussels, Belgium

Full list of author information is available at the end of the article
}

\section{$\triangle B M C$}

(c) The Author(s). 2020 Open Access This article is licensed under a Creative Commons Attribution 4.0 International License, which permits use, sharing, adaptation, distribution and reproduction in any medium or format, as long as you give appropriate credit to the original author(s) and the source, provide a link to the Creative Commons licence, and indicate if changes were made. The images or other third party material in this article are included in the article's Creative Commons licence, unless indicated otherwise in a credit line to the material. If material is not included in the article's Creative Commons licence and your intended use is not permitted by statutory regulation or exceeds the permitted use, you will need to obtain permission directly from the copyright holder. To view a copy of this licence, visit http://creativecommons.org/licenses/by/4.0/ The Creative Commons Public Domain Dedication waiver (http://creativecommons.org/publicdomain/zero/1.0/) applies to the data made available in this article, unless otherwise stated in a credit line to the data. 


\section{Introduction}

Diffuse parenchymal lung diseases (DPLDs) are a heterogeneous group of diseases with a variable amount of fibrosis and inflammation. For prognostic and therapeutic purposes, a precise diagnosis is required. The whole diagnostic process should be performed and discussed within an experienced team during a multidisciplinary discussion (MDD) $[1,2]$. When the diagnosis based on clinical, radiological and biological data is inconclusive, a lung biopsy is recommended [1]. Surgical lung biopsies (SLBs) are currently considered as the gold standard for this purpose as stated in idiopathic pulmonary fibrosis (IPF) guidelines [1].

However, SLB is an invasive procedure with significant comorbidities, a hospitalization for a few days, systematic chest drainage, and a postoperative mortality rate of $2 \%$ up to $3.6 \%$ [3-7]. Therefore, trans-bronchial lung cryobiopsy (TBLC) is increasingly recognized as an alternative technique for the diagnosis of DPLDs [6, 8-11]. TBLC appears to be safer than SLB, and its contribution to the diagnosis obtained via multidisciplinary discussion is comparable to that of SLB, although the histological diagnostic yield is higher with SLB (approximately 80\% for TBLC vs 95\% for SLB) [11].

Interestingly, whereas many data support the use of TBLC for the diagnosis of DPLDs, only three studies compared the result of TBLC and SLB performed in a same patient. A first study was conducted on a limited number of patients (seven) [12] while the other two were conducted on a larger cohort, both providing conflicting results concerning the agreement between TBLC and SLB for histological diagnoses $[13,14]$. Nevertheless, the COLDICE study [14] was the biggest and the unique multicentric study showing a high level of agreement between TBLC and SLB for both histopathological interpretation and MDD diagnoses. In these studies, limited data are available concerning nondiagnostics TBLCs or TBLCs showing a pattern suggestive of a NSIP, two situations in which SLB could particularly provide substantial additional information. Idiopathic NSIP that is nowadays considered as a specific entity [2], is indeed defined histologically by variable proportions of interstitial inflammation and fibrosis with a uniform appearance [15]. However, the assessment of this pathological uniformity is based on the analysis of SLBs that are bigger than TBLCs and performed in different lobes.

Consequently, we hypothesized that the accuracy of NSIP diagnosis obtained on smaller biopsies such as cryobiopsies could be lower than on SLB [9]. NSIP pattern could be mistakenly recognized as other important features could be observed in SLB samples. Particularly, the spatial heterogeneity, important for the diagnosis of UIP patterns, could be missed on TBLCs [16]. This misinterpretation is particularly important as it can have prognostic and therapeutic consequences.
Therefore, the added value of SLB performed after TBLC was evaluated in this study when the latter showed nondiagnostic or unspecific pathological results without confident diagnosis following another multidisciplinary discussion. Preliminary results obtained on a limited number of patients were published in 2017 [9]. In this follow up study, we provide more evidence supporting the benefit of performing SLBs in these selected situations. Moreover, a blinded retrospective analysis of paired TBLCs and SLBs was conducted by three experienced pathologists in ILDs to determine the differences existing between both sampling techniques and to compare the agreement among pathologists for both types of biopsies.

\section{Material and methods \\ Study design}

A multicentric prospective observational study was performed between April 2015 and December 2019 in three Belgian academic hospitals. The study was approved by the ethical committees of the non-leading hospitals and by the leading ethical committee of the Erasme hospital (ref P2015/192). Patients were included if a lung biopsy was indicated and validated by a MDD for the diagnosis of a DPLD. The primary endpoint of the study was to determine the benefit of performing SLBs when TBLCs showed unspecific results (including a pattern suggestive of an NSIP) and without a confident diagnosis following another MDD. Secondary endpoints were to determine the diagnostic yield of TBLC for the diagnosis of DPLDs, to determine the rate of complications related to TBLCs, and to perform a blinded and retrospective review of the paired SLBs and TBLCs samples by the pathologists from the three participating centers. From this retrospective part of the study, the main determinants underlying the hypothesized difference between SLB and TBLC samples will be highlighted, as well as the diagnostic concordance among the three pathologists for both sampling techniques and the level of confidence of the histological diagnosis between paired SLBs and TBLCs samples.

The composition of the multidisciplinary teams includes at least one chest physician, one pathologist, one thoracic radiologist, one specialist in internal medicine or rheumatologist. The patients were informed of the possibility to have a surgical biopsy following the endoscopic procedure in case of equivocal pathological diagnosis or histopathologic pattern suggestive of NSIP. SLBs were obtained by video-assisted thoracoscopic surgery (VATS) and performed in at least two different lobes as recommended in the guidelines for patients with suspected idiopathic pulmonary fibrosis (IPF) [1]. Of note, MDD was conducted in each participation center without blinding, and all indications and results of biopsies (both SLBs and TBLCs) were discussed within the multidisciplinary team. A SLB was not performed in 
patients with a histological NSIP pattern following TBLCs if an associated diagnosis (such as a hypersensitivity pneumonitis) could be made by the MDD with a reasonable probability according to the analysis of all the available data. This includes the HRCT pattern, cellularity of the bronchoalveolar lavage, specific IgG, autoantibodies, drugs, environmental and occupational exposures.

\section{Study population}

Written informed consent for participation in the study was obtained from each patient. Inclusion criteria were the followings: patients at least aged of 18 years old with a pulmonary systolic arterial pressure estimated by echocardiography of less than $40 \mathrm{mmHg}$. Exclusion criteria included the presence of a coagulopathy (platelet count $<100,000 / \mathrm{mm}^{3}$, prothrombin time international normalized ratio - INR $>1.5$, activated partial thromboplastin time - APTT > 35), hypoxemia $\left(\mathrm{PaO}_{2}<55 \mathrm{mmHg}\right.$ on room air) or hypercapnia $\left(\mathrm{PaCO}_{2}>45 \mathrm{mmHg}\right)$, and severe underlying cardiac disease. Collagen vascular disease-associated interstitial lung disease (CVD-ILD) and drug-induced interstitial lung disease (D-ILD) were not formally excluded but efforts were done to avoid lung biopsies in such conditions as the diagnosis can be achieved by other means.

\section{Bronchoscopy and cryobiopsy}

Procedures were performed as previously described [9]. Briefly, all procedures were performed under general anesthesia. We attempted to obtain four biopsies from two different segments of the most affected lobe. For each biopsy, the cryoprobe (1,9 or 2,4 mm, Erbe, Germany) was pushed under fluoroscopic guidance to the distal parenchyma and the probe was withdrawn of 1-2 $\mathrm{cm}$ from the thoracic wall. To control potential severe bleedings, a Fogarty balloon was systematically placed in the lobar bronchus close to the sampled segment, and inflated immediately after biopsy. The bleeding was scored 0 if no bleeding, 1 (mild bleeding) if bleeding stopped with aspiration only and/or insufflation of the Fogarty balloon less than 5 min, 2 (moderate bleeding) if cold saline was used to control the bleeding and/or the Fogarty balloon need to be inflated more than $5 \mathrm{~min}$, and 3 (severe bleeding) if any of the following treatment was required: embolization, selective bronchial intubation, transfusion, admission in an intensive care unit (ICU), or resulting in death or prolonged hospitalization.

\section{Biopsy specimens}

Biopsy specimens were fixed in $10 \%$ formalin and embedded in paraffin. Hematoxylin and eosin as well as Masson's Trichrome, Giemsa, staining were performed as well as immunostaining against pancytokeratins.

\section{Pathological review}

A retrospective histological analysis was performed for patients having both biopsies (TBLCs and SLBs). Three experienced pathologists in DPLDs (MR, FD, and DH) reviewed all biopsies. Biopsies were anonymized (each sample receiving a code number). For each specimen and pathologist, the most likely histological diagnosis and its level of confidence (high, low or very low) were recorded. Histopathological patterns were recognized following the 2018 guideline-refined categories of definite or probable usual interstitial pneumonia, indeterminate for usual interstitial pneumonia, or alternative diagnosis [1]. For some biopsies, no pathological diagnosis could be identified. In that case, the diagnostic confidence was categorized as "not applicable". Histological patterns of probable or indeterminate for UIP were categorized as UIP with a low level of confidence.

Then, a comparison of TBLCs and corresponding SLBs of each patient was performed by each pathologist to evaluate whether the SLB provided additional information as well as to identify the underlying reasons (including the size and the localization of the biopsies). The agreement among pathologists for TBLCs and SLBs diagnoses and the corresponding level of confidence were also determined and compared.

\section{Statistical analysis}

According to the results of the D'Agostino \& Pearson test used to assess the normality of the sample values, simple comparisons between two groups were tested by unpaired Student $t$ tests or Mann-Whitney tests. Proportions were compared using the $\mathrm{Chi}^{2}$ test. Statistical analyses were performed using GraphPad Prism 6 (GraphPad Software, La Jolla, California, USA). For all tests, a $P$-value of less than 0.05 was considered statistically significant.

\section{Results}

TBLCs are useful for the diagnosis of DPLDs

81 patients with TBLCs performed for the diagnosis of a DPLD were included between April 2015 and December 2019. The main clinical characteristics and detailed pathological diagnoses are summarized in the Table 1 . Four TBLCs were performed in the most affected lobe with a mean size of $21 \mathrm{~mm}^{2}$ (ranging from 9 to $44 \mathrm{~mm}^{2}$ ) (Table 1). The preservation of TBLC samples was good to excellent without freezing-related cellular alterations. A specific pathological pattern (excluding a NSIP pattern) was observed in 52 patients $(64 \%)$ whereas undetermined or NSIP patterns were observed in 13 (16\%) and $16(20 \%)$ patients respectively (Fig. 1).

Complications following TBLCs were mainly hemorrhage (mild or moderate in most of the cases) and pneumothoraxes $(21 \%$, half of them requesting a chest 
Table 1 Characteristics of the patients included in the study and having TBLCs performed for the diagnosis of a DPLD

\begin{tabular}{|c|c|c|c|c|c|}
\hline & & $\begin{array}{l}\text { Overall population } \\
(\boldsymbol{n}=81)\end{array}$ & $\begin{array}{l}\text { Specific histological pattern } \\
(\boldsymbol{n}=52)\end{array}$ & $\begin{array}{l}\text { NSIP or nondiagnostic pattern } \\
(\boldsymbol{n}=29)\end{array}$ & $\begin{array}{l}\boldsymbol{p} \\
\text { value }\end{array}$ \\
\hline Gender & Male N (\%) & $40(49)$ & $28(54)$ & $12(41)$ & NS \\
\hline Age, years & Median (range) & $62(26-81)$ & $62(26-81)$ & $64(32-75)$ & NS \\
\hline \multirow[t]{3}{*}{ Smoking history } & Current N (\%) & $11(14)$ & $7(13)$ & $4(14)$ & NS \\
\hline & Former N (\%) & $43(53)$ & $28(54)$ & $15(52)$ & NS \\
\hline & Never N (\%) & $27(33)$ & $17(33)$ & $10(34)$ & NS \\
\hline BMI & Median (range) & $28(17-39)$ & $27(17-39)$ & $29(18-39)$ & NS \\
\hline FVC, \% predicted value & Median (range) & $78(40-134)$ & $78(40-130)$ & $79(41-134)$ & NS \\
\hline $\begin{array}{l}\text { DLCO, \% predicted } \\
\text { value }\end{array}$ & Median (range) & $52(20-85)$ & $52(20-79)$ & $48(28-85)$ & NS \\
\hline \multirow[t]{4}{*}{ HRCT } & Typical UIP N (\%) & $1(1)$ & $1(2)$ & $0(0)$ & NS \\
\hline & Probable UIP N (\%) & $9(11)$ & $5(10)$ & $4(14)$ & NS \\
\hline & $\begin{array}{l}\text { Indeterminate for UIP N } \\
(\%)\end{array}$ & $30(37)$ & $19(37)$ & $11(38)$ & NS \\
\hline & $\begin{array}{l}\text { Alternative diagnostic } \\
\text { N (\%) }\end{array}$ & $41(51)$ & $27(52)$ & $14(48)$ & NS \\
\hline $\begin{array}{l}\text { Number of biopsies/ } \\
\text { patient }\end{array}$ & Mean (range) & $4(1-5)$ & $4(2-5)$ & $4(1-5)$ & NS \\
\hline \multirow[t]{2}{*}{ Size of biopsies (mm2) } & $\begin{array}{l}\text { By specimen Mean } \\
\text { (range) }\end{array}$ & $20,9(9-44)$ & $22(10-44)$ & $18(9-40)$ & 0.049 \\
\hline & $\begin{array}{l}\text { By patient Mean } \\
\text { (range) }\end{array}$ & $76,5(30-152)$ & $82(39-152)$ & $65(30-120)$ & 0.007 \\
\hline
\end{tabular}

Patients were categorized according to the result of the TBLC (specific histological pattern or non-diagnostic/NSIP pattern). Comparisons between groups were made using unpaired Student $\mathrm{t}$ tests or the $\mathrm{Chi}^{2}$ test (proportions). A $P$-value of less than 0.05 was considered statistically significant

drainage) (Table 2). A severe bleeding was present in four patients (5\%) and required at least a prolonged hospitalization and an ICU admission for a 24-h monitoring. No recurrence of bleeding was observed. Whereas the bleeding was controlled in three out of the four patients by a prolonged use of the Fogarty balloon and cold saline solution, one patient required a selective bronchial intubation. In this patient, an acute exacerbation also occurred during the prolonged hospitalization, resulting in an important worsening of the respiratory condition, which required a lung transplantation 2 months after.

Comparison of patient's characteristics between those with nondiagnostic TBLCs and the others showed no differences in term of HRCT pattern distribution but a significant difference in term of size of the cryobiopsies. Indeed, the total surface area per patients of nondiagnostic specimens was smaller than those given a specific diagnosis $\left(65 \pm 24\right.$ vs $82 \pm 27 \mathrm{~mm}^{2}$, mean $\left.\pm \mathrm{SD} ; p<0,01\right)$ (Table 1). We further determined that with a cut-off of a total surface area of $110 \mathrm{~mm}^{2}$, the risk to have nondiagnostic biopsies was lower than 5\%. In line with this observation, two patients had only one TBLC (instead of the attempted four) and none of them had a specific histological diagnosis.
SLB provides critical additional information in case TBLCs are inconclusive or show a pattern suggestive of a NSIP Among the 29 patients without specific diagnosis following TBLCs, seven did not consent to have a SLB (further classified as unclassifiable ILD), and eight received a provisional diagnosis following a novel MDD (5 chronic HP, 2 IPAF, 1 idiopathic NSIP). The reasons for retrieving consent to have a SLB are detailed in supplemental data (Table S1). A SLB was performed for the other 14 patients (six with a suspected NSIP pattern and eight without pathological diagnosis following TBLCs) (Fig. 2). Complications of SLBs within the first 3 months following the biopsies were an acute exacerbation $(n=1)$ and a transient atrial fibrillation $(\mathrm{n}=1)$. No SLB-related death was reported and the median hospitalization time was 5 days (4 days for the chest drainage). As expected, SLB provides ten-fold bigger sample than TBLC $(76 \pm 27$ $\mathrm{mm}^{2}$ for TBLCs vs $676 \pm 307 \mathrm{~mm}^{2}$ for SLB, mean \pm SD) and subpleural area was always present for pathological examination (Supplemental figure, Figure S1). In 11 out of the 14 patients having both TBLC and SLB, a UIP pattern (10 typical and one probable) was observed in the SLB (79\%). Therefore, even if the HRCT pattern was mostly indeterminate or suggestive of another diagnosis in one patient, the final MDD diagnosis was an IPF in 


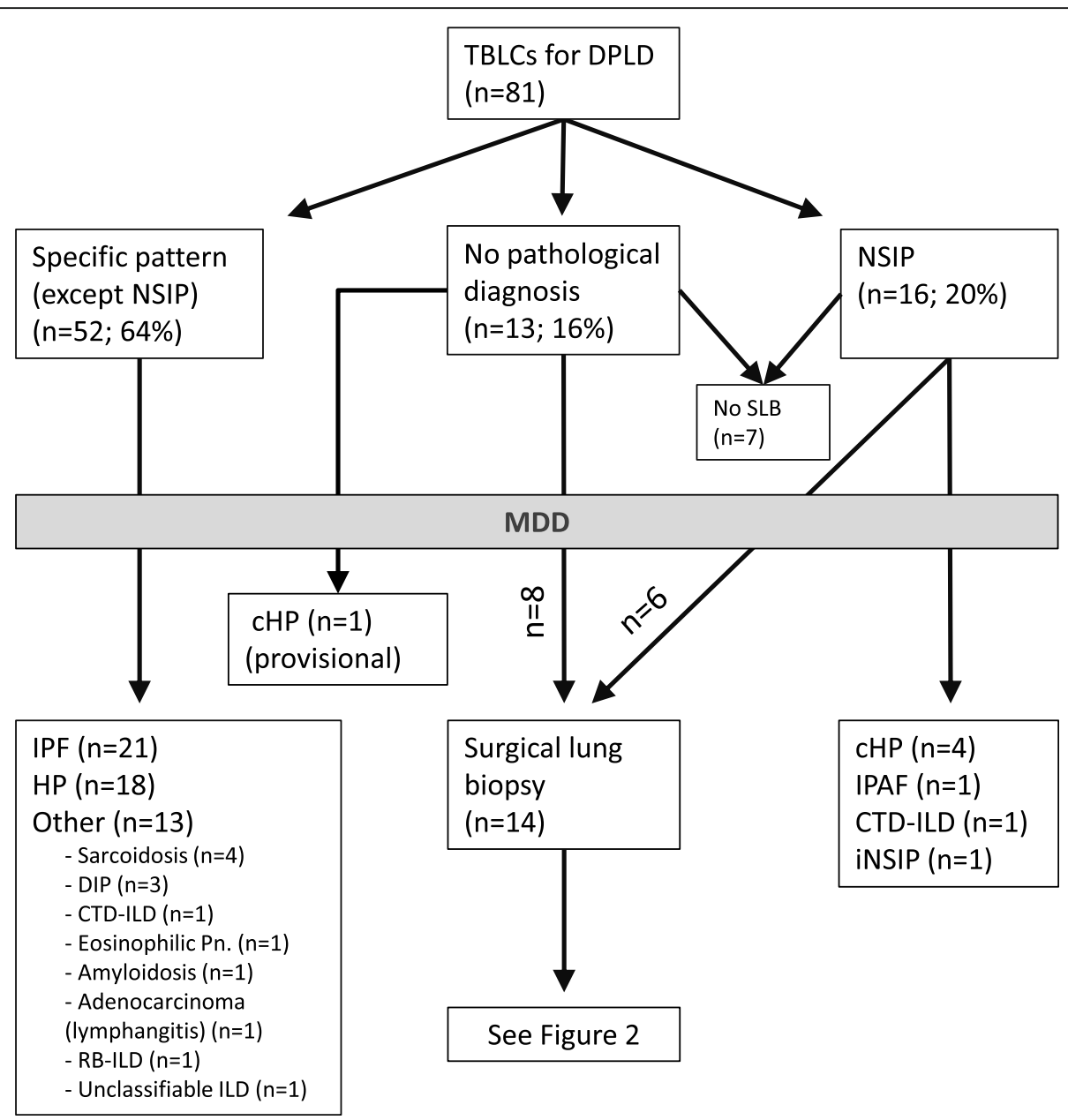

Fig. 1 Flow diagram of the main results obtained for the 81 patients included in the study and having TBLCs for the diagnosis of a DPLD. CHP: chronic hypersensitivity pneumonia; CTD-ILD: connective tissue disease-associated interstitial lung disease; DIP: desquamative interstitial pneumonia; DPLD: diffuse parenchymal lung disease; iNSIP: idiopathic non-specific interstitial pneumonia; IPAF: interstitial pneumonia with autoimmune features; MDD: multidisciplinary discussion; RB-ILD: respiratory bronchiolitis-associated interstitial lung disease; SLB: surgical lung biopsy; NSIP: non-specific interstitial pneumonia; TBLC: trans-bronchial lung cryobiopsy

10 and an IPF "likely" in one patient according to the current guidelines (Fig. 2) [1]. Interestingly, a NSIP pattern was only confirmed in 1 out of the 6 patients with a suspected NSIP on TBLC (17\%), and a HP pattern was observed in two other patients without pathological diagnosis following TBLC. Based on the final MDD taking into account SLB data, the final diagnosis changed in $93 \%$ of the patients having both types of biopsy $(83 \%$ of those with a NSIP pattern on TBLC). In other words, our data showed that if a SLB is performed in a patient with a NSIP pattern on TBLC, another diagnosis will be observed in $83 \%$ of the cases changing significantly the final diagnosis and potentially the subsequent treatment.

Considering patients with specific histological diagnoses provided by the TBLCs, patients with NSIP patterns on TBLCs for which sufficient clinical, biological and radiological data allow the MDD to make a diagnosis without SLBs, and the patient with a NSIP pattern confirmed with both sampling techniques, the diagnostic yield of TBLCs was 74\% (Table 2).

\section{Inter-observer agreement and diagnostic confidence are higher for SLBs than TBLCs}

Three ILDs experienced pathologists further retrospectively and blindly reviewed all biopsies from the 14 patients who benefited from both procedures. Each pathologist was asked to define the most likely diagnosis, even with a low confidence level. Major determinants underlying differences between TBLCs and SLBs were depicted. This analysis demonstrated a higher diagnostic concordance among pathologists for SLB (86\%) compared to TBLCs (43\%) (Fig. 3). Moreover, the level of confidence of the histological diagnosis was much higher for SLBs than for TBLCs (high diagnostic confidence in $88 \%$ of SLB contrasting with low and very low level of confidence in $97 \%$ of TBLCs) (Fig. 3). The benefit to 
Table 2 Complications, diagnostic yield and histological diagnosis after TBLC $(N=81)$

\begin{tabular}{|c|c|c|}
\hline \multirow[t]{4}{*}{ Hemorrhage N (\%) } & Grade 0 & $4(6)$ \\
\hline & Grade 1 (mild) & $47(58)$ \\
\hline & Grade 2 (moderate) & $25(31)$ \\
\hline & Grade 3 (severe) & $4(5)$ \\
\hline \multirow[t]{2}{*}{ Pneumothorax N (\%) } & All & $17(21)$ \\
\hline & Requiring chest drainage & $9(11)$ \\
\hline Diagnostic yield N (\%) & & $60(74)$ \\
\hline \multirow[t]{2}{*}{ Specific histological diagnosis N (\%) } & Including NSIP & $68(84)$ \\
\hline & Excluding NSIP & $52(64)$ \\
\hline \multirow[t]{11}{*}{ Detailed histological diagnosis following TBLCs N (\%) } & UIP & $21(26)$ \\
\hline & $\mathrm{HP}$ & $18(22)$ \\
\hline & NSIP & $16(20)$ \\
\hline & Undetermined & $13(16)$ \\
\hline & Sarcoidosis & $4(5)$ \\
\hline & DIP & $3(4)$ \\
\hline & NSIP/COP overlap & $2(2)$ \\
\hline & Amyloidosis & $1(1)$ \\
\hline & Eosinophilic pn. & $1(1)$ \\
\hline & RB-ILD & $1(1)$ \\
\hline & Adenocarninoma (lymphangitis) & $1(1)$ \\
\hline
\end{tabular}

perform SLB after TBLC in this selected population was not only to improve the diagnostic confidence as it changed the final diagnosis in $60 \%$ of the cases in the retrospective pathological analysis (Table 3). Accordingly, the overall agreement between paired TBLC and SLB for specific histopathological pattern was $40 \%$. When SLBs provide additional information (change in the histological diagnosis and/or an increase in the level of confidence), the major underlying determinants were the size of the biopsies in $90 \%$, a better representation of the subpleural area in $50 \%$ and the localization of the biopsies in different lobes in $23 \%$ of these cases.

Finally, considering the identification of a NSIP pattern on TBLC, the three pathologists retrospectively analyzed the samples of the six patients having TBLCs showing a NSIP pattern and a corresponding SLB. If the concordance between pathologists was perfect to identify a pattern of NSIP, each of them should have identified six NSIP patterns during this retrospective analysis. Surprisingly, there was only an agreement among the pathologists to identify a NSIP pattern for two patients.

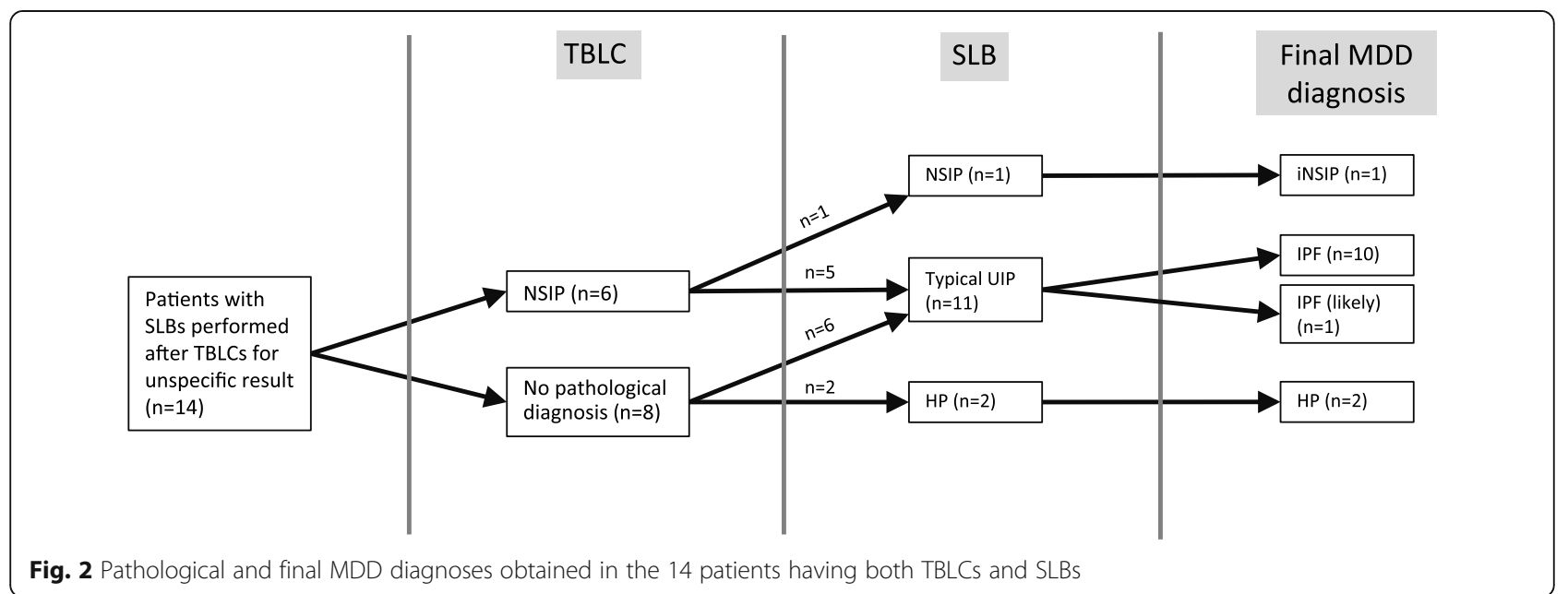



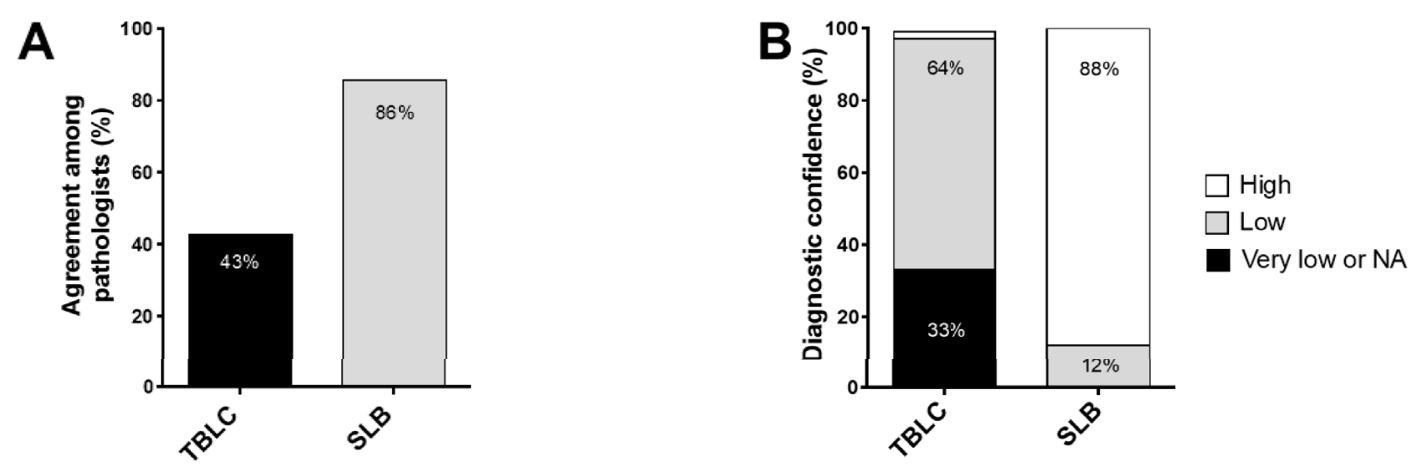

Fig. 3 Comparison between TBLC and SLB regarding the percentage of agreement among pathologists for the most likely diagnosis (a), and the corresponding level of confidence (b). NA: not applicable (non-diagnostic biopsy)

Of note, its identification for any of the pathologist was systematically proposed without a high diagnostic confidence on TBLCs (Table 4). Moreover, the SLBs performed in these two patients only confirmed a NSIP pattern for one pathologist whereas another pathologist proposed a NSIP on SLBs but not on the corresponding TBLCs (Table 4).

\section{Discussion}

Increasing data support the use of TBLCs for the diagnosis of DPLDs with a diagnostic yield ranging between 72 and $87 \%$ [11]. In line with these published results, a diagnostic yield of $74 \%$ was observed from the 81 patients included in this study. The rate of pneumothorax (21\%) was relatively high compare to other reports. This is mostly explained by a high prevalence of fibrotic DPLDs (46\%) known to be associated with higher risk of pneumothorax [10]. Bleeding was mild to moderate in a majority of the cases. There was no TBLC related death. Altogether, our data support that TBLC are safe and globally useful for the diagnosis of DPLD used within MDD. Although no clear standards for adequate sampling in TBLC exist, our data highlight that sample size is of utmost importance. Indeed, non-diagnostic TBLCs were frequently associated with smaller samples. Conversely, if the total surface area of TBLCs was higher than $110 \mathrm{~mm}^{2}$, the risk to have non-diagnostic biopsies was lower than $5 \%$. The number of samples could also influence the frequency of non-diagnostic TBLCs (even if no statistical differences were noticed in our study between the groups). Indeed, more unspecific results were recorded in patients with only one sample. However, the number of patients in this subgroup was extremely low $(n=2)$ to draw definite conclusions.

Beside the growing evidence supporting the use of TBLCs for the diagnosis of DPLDs, only three studies directly compared TBLC and SLB performed in a same patient [12-14]. Romagnoli and colleagues included 21 patients and found a poor agreement for TBLC and SLB histological diagnoses [14]. This result contrasts with data from the recent and bigger COLDICE study including 65 patients and showing a histopathological agreement between TBLC and SLB of 71\%. Moreover, for TBLC with a high to definite confidence at MDD, 95\% of TBLC diagnoses were concordant with SLB diagnoses [13]. In these studies, SLB are performed in all patients whatever the level of confidence obtained after TBLC (5\% of non-diagnostics TBLCs in the COLDICE study).

Table 3 Results of the pathological review of paired TBLCS and SLBs samples by the three experienced pathologists in ILD

\begin{tabular}{|c|c|c|c|c|c|}
\hline & & \multicolumn{3}{|l|}{ Pathologist } & \multirow{2}{*}{$\begin{array}{l}\text { Mean } \\
\text { (SD) }\end{array}$} \\
\hline & & $\overline{M R}$ & $\mathrm{DH}$ & FD & \\
\hline \multicolumn{2}{|c|}{$\begin{array}{l}\text { Change in histological diagnosis following SLB (excluding limited changes in confidence level) } \\
\% \text { (N/Number of patients with TBLC and SLB) }\end{array}$} & $64(9 / 14)$ & $57(8 / 14)$ & $57(8 / 14)$ & $60(4)$ \\
\hline \multicolumn{2}{|c|}{$\begin{array}{l}\text { Additional informations provided by SLB (including changes in confidence level) \% (N/Number } \\
\text { of patients with TBLC and SLB) }\end{array}$} & $93(13 / 14)$ & $71(10 / 14)$ & $79(11 / 14)$ & $81(11)$ \\
\hline \multirow[t]{3}{*}{ Major determinants } & Localisation of the biopsy in different lobes & $23(3 / 13)$ & $10(1 / 10)$ & $36(4 / 11)$ & $23(13)$ \\
\hline & Size of the biopsies & $69(9 / 13)$ & $100(10 / 10)$ & $100(11 / 11)$ & $90(18)$ \\
\hline & Localisation (subpleural vs bronchiolocentric) & $46(6 / 13)$ & $30(3 / 10)$ & $73(8 / 11)$ & $50(22)$ \\
\hline
\end{tabular}

Each pathologist (MR, DH, and FD) provides the most probable histological diagnosis for the 14 patients having both SLBs and TBLCs. For each patient and pathologist, change in histological diagnosis was recorded (row 1) as well as change in histological and/or confidence level (row 2). When SLB provide additional information (including only changes in confidence level), it was asked to the pathologist to describe the main reasons (row 3 ) 
Table 4 Identification of the NSIP pattern by the pathologists

\begin{tabular}{llll}
\hline & Pathologist & & FH \\
\cline { 2 - 4 } & MR & DH & $3 / 14(21 \%)$ \\
\hline NSIP on TBLC & $5 / 14(36 \%)$ & Low (1) & Indeterminate (1) \\
Level of confidence $(n)$ & Indeterminate (2) & Very low (1) & Very low (2) \\
NSIP on SLB & Low (3) & $0 / 14(0 \%)$ & $1 / 14(7 \%)$ \\
NSIP on TBLC and SLB & $1 / 14(7 \%)$ & $0 / 2(0 \%)$ & $0 / 3(0 \%)$ \\
\hline
\end{tabular}

The three experienced pathologists (MR, DH, and FD) retrospectively reviewed samples for which both TBLCs and SLBs were available ( $n=14)$. Recognition of a NSIP pattern was recorded as well as its level of confidence

Moreover, these studies do not specifically address the accuracy of histological NSIP diagnosis in TBLCs compared to SLB (NSIP was only present on TBLC pathological analysis in 2 patients in the COLDICE study and in 3 patients in the Romagnoli study) [13]. In the present study, even if we acknowledge that the number of patients remains low, the benefit to perform SLB after TBLCs (following MDD) was specifically assessed in case of unspecific results including NSIP patterns on TBLC. It was prospectively founded that SLB provides additional information in $93 \%$ of the cases. Our results contrast therefore with those from the COLDICE study in which SLB provides additional information in $23 \%$ of the patients with low confidence or unclassifiable TBLCs diagnoses. Interestingly, NSIP pattern was only confirmed in 1 of the 6 patients with a suspected NSIP pattern on TBLC (17\%) highlighting that a NSIP diagnosis should be questioned if based on TBLCs data only. Even if studies showed that TBLCs affect diagnostic confidence to a similar extend as SLBs within the context of MDD [17], our data showed that SLBs performed after TBLCs do not only increase the diagnostic confidence but also completely change the final diagnosis in most of the cases in this selected population of patients with unspecific TBLCs.

Finally, three pathologists conducted a retrospective and blinded analysis of pathological samples in order to better determine the differences between these two types of biopsies. This analysis does not reflect clinical routine but was interesting as it highlighted a higher interobserver agreement among pathologists with higher confidence levels for SLBs diagnoses compared to TBLCs diagnoses. Moreover, the overall histopathological agreement (independently of the level of confidence) between paired TBLC and SLB was $40 \%$, contrasting to the agreement observed in the COLDICE study of 71\% [13]. Concerning the identification of a NSIP pattern, the diagnostic confidence was systematically low on TBLCs samples and confirmed on SLB in a minority of cases. Determinants underlying additional information from SLBs were analyzed. The bigger size of surgical biopsies was reported in a majority of the cases $(90 \%)$ followed by differences in the localization of the biopsy (subpleural vs bronchiolocentric). Interestingly, the benefit to have samples from different lobes was only noted by the pathologists in only $23 \%$ of the cases.

Important limitations have to be addressed regarding the study. The number of patients having both procedures is small. Hence, additional studies from other groups and with a higher number of patients are required before drawing definite conclusions. In addition, multidisciplinary discussions were not performed blindly and by a unique multidisciplinary team (each of the three participating centers having its own team). Moreover, SLBs were not performed in all patients with TBLCs showing either a NSIP pattern or inconclusive results, representing another possible limitation. This was explained by a proportion of patients who denied having SLBs after TBLCs (24\%) and by patients for which the MDD concluded to a diagnosis with an acceptable level of confidence (this possibility was prespecified in the protocol). Finally, nondiagnostic TBLCs were significantly smaller than those providing a specific diagnosis and the benefit to perform new TBLCs rather than SLBs in those patients was not evaluated.

\section{Conclusions}

Although our study globally supports the role of TBLCs as an alternative to SLB for the multidisciplinary diagnosis of DPLDs, our results highlight the benefit to perform SLB in case of nondiagnostic TBLCs or when a pattern suggestive of a NSIP is observed. Our results also emphasize the importance of adequate sampling technique and sample size to limit the number of TBLCs with unspecific results.

\section{Supplementary information}

Supplementary information accompanies this paper at https://doi.org/10. 1186/s12931-020-01487-w.

Additional file 1: Table S1. Characteristics and main reasons of the seven patients to deny having SLBs after TBLCs. Three of the seven patients experienced a prolonged hospitalization following TBLC (one related to a pneumothorax, one related to a severe bleeding, and one secondary to both a severe bleeding and an acute exacerbation of the underlying ILD). All of the seven patients were afraid of adverse events 
following a SLB, denied to have another general anesthesia and preferred to have a follow up and/or a treatment even if their diagnosis remained uncertain. Of note, except patient \#6 who underwent an acute exacerbation post TBLC, there was no absolute medical contra-indication to perform the SLB in the other six patients.

Additional file 2: Figure S1 Illustration of the bigger size of SLB compared to TBLC. The pleura is also extensively present in the SLB (marked by the black arrow) and conversely most of the time absent in TBLC samples. Hematoxylin and eosin staining.

\section{Abbreviations}

APTT: Activated partial thromboplastin time; BMI: Body mass index; CHP: Chronic hypersensitivity pneumonia; CTD-ILD: Collagen tissue diseaseassociated interstitial lung disease; CVD-ILD: Collagen vascular diseaseassociated interstitial lung disease; D-ILD: Drug-induced interstitial lung disease; DIP: Desquamative interstitial pneumonia; DLCO: Diffusion capacity for carbon monoxide; DPLD: Diffuse parenchymal lung disease; FVC: Forced vital capacity; HP: Hypersensitivity pneumonitis; HRCT: High resolution computed tomography; ICU: Intensive care unit; INR: Prothrombin time international normalized ratio; iNSIP: Idiopathic non-specific interstitial pneumonia; IPAF: Interstitial pneumonia with auto-immune features; IPF: Idiopathic pulmonary fibrosis; MDD: Multidisciplinary discussion; NSIP: Nonspecific interstitial pneumonia; RB-ILD: Respiratory bronchiolitisassociated interstitial lung disease; SLB: Surgical lung biopsy;

TBLC: Transbronchial lung cryobiopsy; UIP: Usual interstitial pneumonia; VATS: Video-assisted thoracoscopic surgery

\section{Acknowledgements}

Not applicable.

\section{Authors' contributions}

$B B, D L, T P, A F, V H, P A$ realized the TBLCs. MR, DH and FD performed the histological examination of the lung biopsies. BB, DL, MR analyzed and interpreted the patient data. BB was a major contributor in writing the manuscript. All authors read, corrected and approved the final manuscript.

\section{Funding}

A grant from Roche and Boehringer Ingelheim was used for the acquisition of the cryobiopsy technology in Erasme Hospital.

\section{Availability of data and materials}

The datasets used and/or analyzed during the current study are available from the corresponding author on reasonable request.

\section{Ethics approval and consent to participate}

The study was approved by the ethical committees of the non-leading hospitals and by the leading ethical committee of the Erasme hospital (ref P2015/192).

\section{Consent for publication}

Not applicable.

\section{Competing interests}

$\mathrm{BB}$ received a grant from Roche and Boehringer Ingelheim to support this study. Other authors disclose no conflict of interest in relation to this manuscript.

\section{Author details}

'Department of Pneumology, Hôpital Erasme, Université libre de Bruxelles, 808 route de Lennik, 1070 Brussels, Belgium. '2Department of Pneumology, Cliniques Universitaires Saint-Luc, Université Catholique de Louvain, Brussels, Belgium. ${ }^{3}$ Department of Pneumology, Centre Hospitalier Universitaire de Liège, Liège, Belgium. ${ }^{4}$ Department of Pneumology, AZ Glorieux, Ronse, Belgium. ${ }^{5}$ Department of Pathology, Cliniques Universitaires Saint-Luc, Université Catholique de Louvain, Brussels, Belgium. ${ }^{6}$ Department of Pathology, Centre Universitaire de Liège, Liège, Belgium. ${ }^{7}$ Department of Pathology, Hôpital Erasme, Université libre de Bruxelles, Brussels, Belgium.
Received: 1 May 2020 Accepted: 16 August 2020

Published online: 04 September 2020

\section{References}

1. Raghu G, Remy-Jardin M, Myers JL, et al. Diagnosis of idiopathic pulmonary fibrosis. An official ATS/ERS/JRS/ALAT clinical practice guideline. Am J Respir Crit Care Med. 2018;198(5):e44-68.

2. Travis WD, Costabel U, Hansell DM, et al. ATS/ERS committee on idiopathic interstitial pneumonias. An official American Thoracic Society/European Respiratory Society statement: update of the international multidisciplinary classification of the idiopathic interstitial pneumonias. Am J Respir Crit Care Med. 2013;188(6):733-48.

3. Han Q, Luo Q, Xie JX, et al. Diagnostic yield and postoperative mortality associated with surgical lung biopsy for evaluation of interstitial lung diseases: a systematic review and meta-analysis. J Thorac Cardiovasc Surg. 2015;149(5):1394-401.

4. Morris D, Zamvar V. The efficacy of video-assisted thoracoscopic surgery lung biopsies in patients with Interstitial Lung Disease: a retrospective study of 66 patients. J Cardiothorac Surg. 2014;9:45.

5. Sonobe M, Handa T, Tanizawa K, et al. Videothoracoscopy-assisted surgical lung biopsy for interstitial lung diseases. Gen Thorac Cardiovasc Surg. 2014; 62(6):376-82.

6. Ravaglia C, Bonifazi M, Wells AU, et al. Safety and diagnostic yield of transbronchial lung cryobiopsy in diffuse parenchymal lung diseases: a comparative study versus video-assisted thoracoscopic lung biopsy and a systematic review of the literature. Respiration. 2016:91(3):215-27.

7. Hutchinson JP, McKeever TM, Fogarty AW, Navaratnam V, Hubbard RB. Surgical lung biopsy for the diagnosis of interstitial lung disease in England: 1997-2008. Eur Respir J. 2016;48(5):1453-61.

8. Hetzel J, Maldonado F, Ravaglia C, et al. Transbronchial cryobiopsies for the diagnosis of diffuse parenchymal lung diseases: expert statement from the Cryobiopsy Working Group on Safety and Utility and a Call for Standardization of the Procedure. Respiration. 2018;95(3):188-200.

9. Bondue $B$, Pieters $T$, Alexander $P$, et al. Role of transbronchial lung cryobiopsies in diffuse parenchymal lung diseases: interest of a sequential approach. Pulm Med. 2017;2017:6794343.

10. Casoni GL, Tomassetti S, Cavazza A, et al. Transbronchial lung cryobiopsy in the diagnosis of fibrotic interstitial lung diseases. PLoS One. 2014;9(2): e86716.

11. Maldonado F, Danoff SK, Wells AU, et al. Transbronchial cryobiopsy for the diagnosis of interstitial lung diseases: CHEST guideline and expert panel report. Chest. 2019;157(4):1030-42.

12. Zaizen $Y$, Kohashi $Y$, Kuroda $K$, et al. Concordance between sequential transbronchial lung cryobiopsy and surgical lung biopsy in patients with diffuse interstitial lung disease. Diagn Pathol. 2019;14(1):131.

13. Troy LK, Grainge C, Corte TJ, et al. Diagnostic accuracy of transbronchial lung cryobiopsy for interstitial lung disease diagnosis (COLDICE): a prospective, comparative study. Lancet Respir Med. 2020;8(2):171-81.

14. Romagnoli M, Colby TV, Berthet JP, et al. Poor concordance between sequential transbronchial lung cryobiopsy and surgical lung biopsy in the diagnosis of diffuse interstitial lung diseases. Am J Respir Crit Care Med. 2019;199(10):1249-56.

15. Travis WD, Hunninghake G, King TE Jr, et al. Idiopathic nonspecific interstitial pneumonia: report of an American Thoracic Society project. Am J Respir Crit Care Med. 2008;177(12):1338-47.

16. Flaherty KR, Travis WD, Colby TV, et al. Histopathologic variability in usual and nonspecific interstitial pneumonias. Am J Respir Crit Care Med. 2001; 164(9):1722-7.

17. Tomassetti $\mathrm{S}$, Wells $\mathrm{AU}$, Costabel U, et al. Bronchoscopic lung cryobiopsy increases diagnostic confidence in the multidisciplinary diagnosis of idiopathic pulmonary fibrosis. Am J Respir Crit Care Med. 2016;193:745-52.

\section{Publisher's Note}

Springer Nature remains neutral with regard to jurisdictional claims in published maps and institutional affiliations. 